\title{
CIFUENTES HONRUBIA, J. L. (2018). CONSTRUCCIONES CON CLÍTICO FEMENINO LEXICALIZADO. MADRID: VERBUM. 422 PÁGINAS
}

\author{
JULio TORRES SOlER \\ Universidad de Alicante \\ jts30@alu.ua.es \\ https://orcid.org/0000-0003-4866-6605
}

Este libro de José Luis Cifuentes Honrubia aborda las construcciones lexicalizadas en las que aparece un pronombre clítico femenino acusativo que no muestra ningún antecedente para el complemento directo, como arreglárselas o fastidiarla. Para averiguar las razones que han motivado la presencia del clítico, el autor lleva a cabo un estudio histórico de 99 construcciones de este tipo, convencido de poder encontrar en la historia de la lengua las claves de su fijación, como él mismo confiesa en el prólogo. El resultado es una descripción amplia de estas construcciones en su diacronía, que atiende a cuestiones sintácticas, semánticas y pragmáticas.

El libro consta de tres capítulos: en primer lugar, una gran introducción teórica; a continuación, la descripción histórica de las construcciones con clítico femenino, que constituye el fundamento del trabajo, y por último, las conclusiones. En el capítulo I, la "introducción”, se presentan y se discuten algunas teorías lingüísticas que más tarde se utilizan en la descripción de las locuciones verbales. En primer lugar, se introducen algunas consideraciones que se han hecho desde la fraseología sobre estas construcciones. Se dedica una especial atención a la propuesta de Delbeque (1997), quien explica el predominio del clítico femenino sobre el masculino en este tipo de construcciones porque, al ser el femenino el género marcado en español, se asocia con usos menos previsibles. Según la autora, esto favorece la identificación del uso no habitual de la construcción y facilita el proceso de lexicalización. De forma semejante, la morfología plural del clítico como elemento marcado propicia particularmente la interpretación del significado contextual de la construcción.

A continuación, se revisan los diferentes esquemas construccionales de este tipo de locuciones: 1) verbo transitivo + clítico; 2) verbo transitivo + clítico + complemento indirecto; 3) verbo pronominal + clítico y 4) verbo intransitivo + clítico. Sobre este último esquema, en el que se incluyen las locuciones pirárselas, guillárselas, etc., se comenta que es especialmente interesante la presencia de un clítico de complemento directo con un verbo

Para citar esta reseña / To cite this book review: Rodríguez Faneca, Cristina (2020). Reseña de González-Fernández, Adela y Rodríguez-Tapia, Sergio (eds.) (2019). Estudios lingüísticos en torno al turismo: terminología, cultura y usuarios. Valencia: Tirant Humanidades. 290 Páginas. ELUA, 34: 241-247. https://doi.org/10.14198/ELUA2020.34.15

Enlace / Link: https://doi.org/10.14198/ELUA2020.34.15

(c) BY-NC Este trabajo se publica bajo una licencia de Creative Commons Reconocimiento-NoComercial 4.0 Internacional 
intransitivo. Además, se reconoce que estas construcciones admiten numerosas variaciones mediante diversas marcas sintácticas, que a menudo permiten diferenciar entre los posibles significados contextuales de las locuciones. En las siguientes páginas, se discute el posible valor eufemístico de las locuciones con clítico femenino. Tras revisar la definición de eufemismo de la tradición discursiva, se descarta que las construcciones con clítico femenino constituyan un caso de sintaxis eufemística, argumentando que el uso de estas construcciones no mitiga la carga negativa de las expresiones.

Una vez revisadas las consideraciones hechas sobre las locuciones con clítico femenino hasta el momento, y habiendo descartado un posible análisis eufemístico, se dedica el resto de apartados de la introducción a presentar el modelo teórico que verdaderamente constituye la base del análisis posterior de estas construcciones: la teoría de la subjetivación. Para ello, se revisa de forma sintética y completa la noción de subjetivación, así como sus derivaciones más recientes y su relación con otros mecanismos de cambio lingüístico, ofreciendo una introducción clara y precisa para aquellos lingüistas que no estén familiarizados con esta línea de investigación. En primer lugar, se describe la propuesta de Elisabeth Closs Traugott (1982, 1989, 1995, 1996, 2011, 2016), quien entiende la subjetivación como un mecanismo semántico-pragmático que tiene lugar en diacronía, por el cual los significados pasan de referirse a la realidad externa para expresar la visión subjetiva del hablante hacia lo que se dice. También se explica la noción de intersubjetivación, desarrollada por la misma autora, que consiste en el mecanismo por el cual los significados, ya subjetivizados, pasan a codificar significados interpersonales, es decir, que expresan la atención del hablante hacia la imagen del interlocutor. Seguidamente, se compara la teoría de la subjetivación de Traugott con las propuestas de otros autores, como Langacker (1990, 1993, 1999), Sanders y Spooren (1996), Verhagen (2005), Schiffrin (1990), Nuyts (2001) y Narrog (2012, 2014, 2017). Debido a su trascendencia, se lleva a cabo con particular detenimiento la comparación con la propuesta de Langacker, para quien la subjetivación es un fenómeno sincrónico que tiene que ver con la presencia implícita de la perspectiva del hablante en la escena conceptual, codificada en una construcción. Pese a las diferencias, se concluye que ambas visiones de la subjetivación son complementarias.

A continuación, se incide en las consecuencias sintácticas de la subjetivación. Siguiendo a Company (2004), se explican tres efectos sintácticos: a) el debilitamiento o pérdida del control que ejerce el agente sobre evento; b) la ampliación del alcance de la predicación y c) la fijación, aislamiento y autonomía predicativa del elemento. En definitiva, la subjetivación conlleva un empobrecimiento sintáctico que compensa el fuerte enriquecimiento pragmático. Esta idea lleva a discutir la relación entre la subjetivación y la pragmatización. Cifuentes Honrubia, que parte de una concepción de la sintaxis, la pragmática y la semántica como dominios no estrictamente separables, no considera necesario emplear el concepto de pragmatización en su trabajo.

En las siguientes páginas, se presentan los planteamientos esenciales de la Gramática de Construcciones y se introducen algunas de sus aportaciones al estudio diacrónico de la gramática, como la idea de que los elementos lingüísticos no cambian solos o aislados, sino ubicados en construcciones o distribuciones sintagmáticas específicas. Se analiza con particular detenimiento la propuesta de Traugott y Trousdale (2013) sobre la construccionalización, entendida como el proceso diacrónico de creación de nuevas combinaciones de signos con forma y significado nuevos. La construccionalización es descrita como un proceso gradual 
en el que se distinguen algunos micropasos, que el autor se detiene a explicar: a) innovación y reanálisis, b) reutilización, c) inferencia invitada, d) convencionalización, e) reorganización y f) otros cambios. Por último, se señala que la construccionalización conlleva cambios en la productividad, la esquematicidad y la composicionalidad de la construcción.

El último apartado de este capítulo está dedicado a explorar el papel de la analogía en el cambio lingüístico, ya que constituye una noción fundamental en el análisis posterior de las construcciones con clítico femenino lexicalizado. Es importante la distinción que se hace entre pensamiento analógico, entendido como la capacidad cognitiva del ser humano para comparar dos realidades, transferir información de un dominio cognitivo a otro y extraer generalizaciones, y la analogización, entendida como el mecanismo de cambio lingüístico motivado por el pensamiento analógico por el que asignamos una nueva forma o significado a una unidad lingüística. A continuación, se estudia el papel de la analogía en los procesos de gramaticalización. Se indica que la analogía está implicada en la gramaticalización al menos de dos formas: por un lado, puede afectar a algunos rasgos del elemento que se gramaticaliza y, por otro lado, puede producir un aumento de la productividad del nuevo esquema gracias a la atracción de nuevas unidades. Por último, se reconoce que la analogía puede producirse a través de modelos o esquemas abstractos que favorecen la productividad de una determinada estructura.

En el capítulo II, llamado "construcciones con clítico femenino lexicalizado", se analizan, una por una, 99 locuciones con clítico femenino. Para ello, se aportan datos y ejemplos extraídos del CORDE y del CREA, que contribuyen a configurar una visión histórica de cada construcción, y se discuten las claves de su fijación. En el primer apartado de este capítulo se analizan las locuciones hacerla, jugársela, pringarla, joderla, pifiarla, cagarla, fastidiarla, jorobarla y jeringarla. Hacerla, que es la primera construcción con clítico femenino lexicalizado atestiguada, indica una acción negativa que queda implicada contextualmente. Teniendo en cuenta el valor de la proforma hacer, se discute un posible valor eufemístico en su origen, que pronto habría desaparecido. En cuanto a jugársela a alguien, que se refiere a un engaño, su motivación podría encontrarse en contextos de juego en los que la referencia implicada es jugada o partida. En el caso de pringarla, joderla, pifiarla, cagarla, fastidiarla, jorobarla y jeringarla, todas estas construcciones indican una acción negativa realizada por el propio sujeto y que incide en él mismo, por lo que se trata de errores o faltas. La motivación de estas construcciones se explica, por un lado, por la influencia analógica que han podido ejercer unas sobre otras, que es especialmente notoria en el caso de joderla sobre jorobarla y jeringarla y, por otro lado, por diversos cambios semánticos experimentados por los verbos. En todos los casos, el referente del clítico femenino es la propia acción del sujeto.

En el segundo apartado, se analizan las locuciones guardársela, pagársela, debérsela, jurársela, ganársela y cargársela. Por un lado, en guardársela y pagársela el clítico hace referencia semántica a algún tipo de ofensa o agravio, mientras que los verbos mantienen su significado habitual. En Debérsela y jurársela, el clítico también hace referencia semántica a una ofensa o mala acción, pero en este caso la locución indica algún tipo de venganza debida a dicha ofensa. El autor encuentra muchos casos en que estas cuatro últimas locuciones están vinculadas en el uso, lo que hace probable una influencia analógica entre ellas. Aunque mucho más reciente, ganársela está relacionada con las anteriores, pero en este caso el clítico hace referencia semántica a un castigo, motivado igualmente por alguna mala acción u 
ofensa. Cargársela es algo distinta al resto de locuciones, puesto que el sujeto se ve afectado por la acción. El referente semántico del clítico es una carga, entendida metafóricamente como cualquier tipo de mala acción, por la que el sujeto recibe un castigo.

Las locuciones gozarla, correrla y mangarla, descritas en el tercer apartado, presentan cada una un proceso de fijación diferente. En el caso de gozarla, históricamente el clítico podría tener varios antecedentes, especialmente la mujer, la vida y la ocasión. Posteriormente, la locución queda fijada con el significado 'pasarlo bien, disfrutar', sin que el clítico encuentre referencia sintáctica alguna. En cuanto a correrla, que se refiere a acciones potencialmente peligrosas llevadas a cabo con cierta diversión, el autor discute diversos posibles antecedentes históricos del clítico y argumenta que el más probable es el sustantivo correría, en su significado 'andanza o aventura'. La locución mangarla, de uso muy limitado y socialmente marcada como gitanismo, significa 'vivir sin hacer nada'. El significado se origina por metonimia a partir del verbo mangar 'pedir, mendigar'. En cuanto al clítico, el autor considera que no encuentra referente sintáctico ni semántico, y afirma que su presencia podría deberse a la analogía con otras locuciones que presentan un contexto de uso similar.

En el cuarto apartado del capítulo se analizan las locuciones entendérselas, habérselas, campaneárselas, avenírselas, sabérselas, ingeniárselas, prometérselas, buscárselas, componérselas, arreglárselas, amañárselas, pintárselas, manejárselas, gobernárselas, bandeárselas, agenciárselas, apañárselas y olérselas. Entre estas locuciones, entendérselas es un caso particularmente interesante porque es muy antigua e influye en gran medida en muchas otras locuciones con clítico femenino, como avenírselas, ingeniárselas, buscárselas y componérselas. Además, el autor describe una serie de cambios semánticos y construccionales por los que entendérselas pasa de tener como referente semántico las mañas o astucias de otra persona hasta presentar un papel activo del sujeto con el significado 'desenvolverse bien'. Estas locuciones presentan un esquema construccional muy productivo en español y la mayoría de ellas están vinculadas al significado 'desenvolverse adecuadamente'.

El quinto apartado aborda las locuciones tomarlas, afufarlas, apeldarlas, liarlas, volarlas, guillárselas, tocárselas, pirárselas y emprenderla. De todas las locuciones anteriores, que tienen en común su significado de desplazamiento, tomarlas es la más antigua. Se argumenta que el origen de tomarlas se encuentra en tomar las calzas de Villadiego. A partir de esta expresión, que significaba 'desplazamiento', se crea tomarlas, cuya vinculación con las calzas de Villadiego siempre habría estado presente en la mente de los hablantes. Pese a que tomarlas tiene una corta vida en la historia del español, se defiende su influencia analógica sobre otros verbos de desplazamiento que lexicalizan el clítico femenino como una marca de expresividad, dando lugar a las locuciones afufarlas, apeldarlas, liarlas, volarlas, guillárselas, tocárselas y pirárselas. Diferente es el caso de emprenderla, que también expresa la realización de un viaje o el recorrido de un camino, pero cuya lexicalización, en este caso, se explica por la correferencialidad que antiguamente el clítico encontraba en elementos como viaje, navegación o marcha.

En el sexto apartado, se estudian las locuciones tenerlas, habérselas, pegarla, emprenderla, tomarla, vérselas, agarrarla, liárselas y cogerla. Todas estas locuciones tienen en común, por un lado, que su significado está relacionado con un enfrentamiento o una pelea $\mathrm{y}$, por otro lado, que se combinan con un elemento preposicional introducido por la preposición con. El caso de habérselas con alguien es particularmente interesante porque, en primer lugar, se atestigua con la forma haberlo con alguien. Se argumenta que más tarde 
la locución adopta el clítico femenino plural por analogía con tenerlas con alguien. En el séptimo apartado se analizan las locuciones pelárselas, cantarlas y piarlas. En cuanto a pelárselas, se defiende que el clítico tuvo como referente en un primer momento las barbas. La expresión pelarse las barbas, cuyo origen reside en el acto de tirarse de las barbas por el enfado, dio su significado de 'enfado' a la locución pelárselas, que más tarde se amplía metafóricamente a cualquier acción 'con gran intensidad o vehemencia', significado que llega hasta la actualidad. Tanto cantarlas como piarlas indican la expresión de una opinión negativa. El autor argumenta que la fijación de cantarlas se produce a partir de cantar las cuarenta, que se documenta en el contexto del juego del tute, mientras que la fijación de piarlas se produce por analogía con otras locuciones, en particular con cantarlas.

En el octavo apartado del capítulo se abordan las locuciones pegársela, clavársela, colársela, dársela, diñársela, metérsela, freírsela y mamarla, todas ellas vinculadas con la idea de engañar. En cuanto a pegársela, se argumenta que en su fijación queda implicada la idea de pega, con su significado 'engaño', sustantivo con el que guarda una importante relación semántica y morfológica. Pegársela ejerce una influencia analógica que da lugar a la fijación de dársela, y a su vez, dársela influye analógicamente sobre diñársela. En el caso de colársela, se explica que su fijación viene dada por el propio significado del verbo y que el clítico encuentra como referente semántico una mentira o un engaño. La fijación de clavársela ocurre a partir de contextos en los se encuentra como referente del clítico espada $\mathrm{u}$ otros objetos punzantes, mientras que en la fijación de meterla queda implicado en un primer momento el órgano sexual masculino. Respecto a la fijación de freírsela y mamarla, se discuten posibles motivaciones y contextos, pero los ejemplos encontrados son demasiado escasos como para llegar a conclusiones convincentes.

En el noveno apartado se tratan las locuciones echárselas, tirárselas, dárselas y pintarla. En cuanto a echárselas, que significa 'jactarse', se propone una relación con la expresión echar de la gloriosa, que explicaría su combinación con sintagmas preposicionales encabezados por de. Además, debido a su semejanza semántica, echárselas ejerció una influencia analógica que motivó la fijación de tirárselas y dárselas, aunque en esta última habría quedado implicado además el sustantivo muestras. En el caso de pintarla, se discute la evolución semántica del verbo, cuyo significado 'exagerar o engrandecer una situación verbalmente' es adoptado por pintarla cuando, por analogía con otras construcciones con clítico femenino, la locución queda fijada. El décimo apartado aborda las locuciones armarla, liarla y montarla. La lexicalización de armarla y de montarla se explica por la implicación contextual del referente del clítico, que es una mala acción, habitualmente un alboroto o un trastorno. En cuanto liarla, es importante su relación con armar un lío, que habría facilitado que armarla influyera por analogía en la fijación de liarla.

En el undécimo apartado se analizan gastarlas y traérselas, que indican un comportamiento inhabitual o malicioso. Se argumenta que gastarlas se lexicalizó a partir de contextos en los que el referente del clítico era broma u otros sustantivos similares como chanza o jugarreta. En cuanto a traerlas, se plantea la posibilidad de que el referente del clítico fuese, en el origen de la construcción, el sustantivo armas. A partir de ese contexto, el significado se habría desplazado metonímicamente desde aquello que se desplaza hasta la actividad propia de ese objeto. En la duodécima sección se estudian las locuciones dormirla, cogerla, pillarla, agarrarla y engancharla. En todas estas construcciones, el clítico hace referencia semántica al concepto de borrachera, tal como demuestra el autor. Se trata, por tanto, de 
construcciones en las que el referente del clítico quedó contextualmente implicado, pasando a integrarse en el significado de la propia construcción. También se aprecia una influencia analógica, especialmente de cogerla sobre pillarla, agarrarla y engancharla.

El decimotercer apartado se ocupa de las construcciones tragárselas, pasarlas y envainársela. Tragárselas, que significa 'aceptar cosas que son inaceptables', se lexicaliza cuando el referente del clítico, que son las mentiras, engaños ofalsedades, queda implicado en el significado del verbo. En cuanto a pasarlas, se argumenta que el origen de la construcción se encuentra en algunas expresiones en las que el elemento vivencias está implicado, en especial pasar las de Caín. En el caso de envainársela, el clítico originariamente tiene como claro referente la espada. Desde la acción de envainar la espada, el significado de la construcción evolucionó metafóricamente a 'rectificar'. En el siguiente apartado se abordan las locuciones entregarla, diñarla, espicharla, palmarla, pringarla y cascarla, que significan todas ellas 'morir'. En el caso de entregarla, el referente implicado del clítico es el alma, la vida o algún sustantivo equivalente, al igual que sucede probablemente en diñarla, gitanismo en el que entregarla podría haber influido analógicamente. En cuanto a espicharla, palmarla, pringarla y cascarla, todos ellos desarrollan metafórica o metonímicamente el significado 'morir' independientemente del clítico, que podría haber aparecido por analogía con el resto de construcciones con clítico femenino lexicalizado.

El decimoquinto apartado se centra en traérsela, refanfinfársela, pelársela, bufársela, sudársela y soplársela. En todas estas locuciones el clítico femenino tiene como referente el miembro sexual masculino y todas ellas expresan indiferencia. Tráersela, que se combina con floja o al pairo, alude a la ausencia de erección, desde donde metafóricamente se llega al significado actual 'indiferencia', como ocurre también en refanfinfársela y bufársela. En cuanto a pelársela, sudársela y soplársela, se relacionan con la masturbación o con la felación, y en los tres casos, la relación con el significado 'indiferencia' se explica por una actitud machista de desprecio hacia quien realiza dichas prácticas a otra persona, pasando del desprecio a la indiferencia. En el decimosexto y último apartado se estudian seis locuciones, deparársela, matarlas, cazarlas, saltarla y chocarla, que no guardan una relación clara entre sí ni con otras en particular. Deparársela es en realidad una frase proverbial, Dios te la depare buena, en que el clítico hacía referencia a receta. En cuanto a matarlas, que se combina con callando, el autor defiende que el clítico hace alusión genérica a una acción negativa que se realiza sigilosamente. Cazarlas, que se combina con al vuelo, hace referencia a tomar y hacer propias las ideas o pensamientos. En cuanto saltarla, que aparece en la expresión andar a la que salta, se plantean dos posibles contextos originarios, la caza y el juego. Por último, chocarla es el resultado de la subjetivación de la mano, desde donde se pasa a significar 'conformidad'.

Una vez analizadas de forma individual todas las construcciones con clítico femenino lexicalizado, en el capítulo III, "Conclusiones", se lleva a cabo una recapitulación de las ideas principales del análisis de cada grupo de locuciones aplicando nociones teóricas como la subjetivación, la construccionalización y la analogía, entre otras. De forma general, se afirma que las construcciones con clítico femenino lexicalizado son un tipo específico de construcción en español que sirve para marcar ciertos eventos como negativos y mostrar cercanía con el interlocutor. Por último, se distinguen algunos puntos en común en el proceso de construccionalización de estas locuciones. En todos los casos, el complemento directo se ha subjetivado, es decir, la referencia del clítico femenino queda implicada contextualmente 
y ha pasado a formar parte del significado convencional de la construcción. El segundo paso del proceso de construccionalización de las locuciones, muy habitual pero no imprescindible, es un cambio de significado del evento. El tercer y último paso, tampoco necesario, es la analogía, que facilita que otras construcciones con significados semejantes adopten el clítico femenino. Se afirma, por tanto, que la analogía cumple un papel fundamental en la productividad de estas construcciones.

Por último, en el apartado "Anexos", se incluye una tabla que recoge de forma esquemática algunos datos de las construcciones con clítico femenino estudiadas: el periodo de vigencia, el elemento subjetivado, el elemento que ejerce una influencia analógica específica y el cambio de significado que experimenta la construcción. A continuación, se incluye un índice de construcciones y expresiones que han sido consideradas a lo largo del análisis de las construcciones con clítico femenino lexicalizado.

En definitiva, este volumen monográfico de José Luis Cifuentes Honrubia constituye una aportación importante al conocimiento de las locuciones con clítico femenino en español. Además, el estudio de estas construcciones se hace desde una perspectiva teórica que incorpora al análisis de la lengua española algunas nociones de reciente desarrollo, como la subjetivación y la construccionalización. Por tanto, este volumen es una referencia imprescindible para aquellos interesados en la historia, la motivación o los cambios semánticos de las locuciones con clítico femenino, pero también para quienes deseen una lectura actualizada sobre los mecanismos de cambio lingüístico en la interfaz entre la sintaxis, la semántica y la pragmática. 
